\title{
Bray John, Maurer Petra \& Butcher Andrea (eds), Ladakh. Historical Perspectives and Social Change
}

2015, The Tibet Journal 40(2), numéro spécial, 283 pages

\section{Patrick Kaplanian}

\section{(2) OpenEdition}

\section{Journals}

Édition électronique

URL : https://journals.openedition.org/emscat/2950

DOI : 10.4000/emscat.2950

ISSN : 2101-0013

Éditeur

Centre d'Etudes Mongoles \& Sibériennes / École Pratique des Hautes Études

Référence électronique

Patrick Kaplanian, « Bray John, Maurer Petra \& Butcher Andrea (eds), Ladakh. Historical Perspectives and Social Change ", Études mongoles et sibériennes, centrasiatiques et tibétaines [En ligne], 48 | 2017, mis en ligne le 05 décembre 2017, consulté le 13 juillet 2021. URL : http://journals.openedition.org/ emscat/2950; DOI : https://doi.org/10.4000/emscat.2950

Ce document a été généré automatiquement le 13 juillet 2021.

(c) Tous droits réservés 


\title{
Bray John, Maurer Petra \& Butcher Andrea (eds), Ladakh. Historical Perspectives and Social Change
}

\author{
2015, The Tibet Journal 40(2), numéro spécial, 283 pages
}

\section{Patrick Kaplanian}

\section{RÉFÉRENCE}

Bray John, Maurer Petra \& Butcher Andrea (eds), Ladakh. Historical Perspectives and Social Change, 2015, The Tibet Journal 40(2), numéro spécial

1 Ce numéro spécial de la revue The Tibet journal constitue en fait une partie des actes du $16^{\mathrm{e}}$ colloque de l'IALS (International Association for Ladakh Studies) qui s'est tenu à Heidelberg en avril 2013. Les articles qui le composent suivent un ordre chronologique.

2 Le titre de l'article de Christian Faggionato, "The History of dge lugs pa in the Nubra Valley ", est trop modeste car son sujet l'amène à nous présenter l'histoire d'ensemble de la vallée, en insistant sur les aspects religieux. La vallée de la Nubra se situe au nord de Leh, l'ancienne capitale royale du Ladakh, aujourd'hui chef-lieu de district. On y accède par un col à $5380 \mathrm{~m}$, sur une route goudronnée considérée comme la plus haute du monde. Cet isolement relatif en fait un monde un peu à part. L'auteur note que, comme dans le reste du Ladakh, mais contrairement au Tibet, les monastères n'ont jamais cherché à exercer le pouvoir, le laissant aux chefs locaux et aux représentants de la monarchie de Leh. Il s'appuie sur une importante documentation rassemblée sur place et qui lui a servi à écrire sa thèse (Faggionato 2013).

Le Zanskar est une haute vallée enclavée au sud de Leh. Encore plus isolé que la Nubra, il était, avant sa conquête par le roi du Ladakh au XvIII siècle, divisé entre deux petits royaumes, Padum et Zangla. Rob Linrothe s'intéresse à un chorten dit " de la reine " dans le village de Karsha qui faisait partie du royaume de Zangla : un monument ignoré aussi bien des spécialistes que de la population locale qui s'en servait de bergerie ou de 
cuisine. Or ce chorten, que Linrothe date du dernier quart du Xvie siècle, est couvert de fresques. L'intérêt de l'article est moins dans l'ajout de nouvelles fresques à un catalogue ladakhi déjà bien fourni que dans les thèmes iconographiques de ces fresques: on y trouve à la fois des thèmes drukpa ('brug pa) et gelukpa (dge lugs pa). L'auteur explique cela par une tolérance des rois et des nobles du royaume de Zangla vis-à-vis de ces deux courants du bouddhisme tibétain. L'article est abondamment illustré avec un commentaire détaillé de certaines fresques.

Petra Maurer s'intéresse à Nawang Tsering (sgrub chen ngag dbang tshe ring, 1657-1734) le célèbre yogi du Zanskar'1. Elle est frappée par la présence d'un chapitre inhabituel dans sa biographie : dans son enfance le futur yogi fut persécuté par sa bellemère. Ceci est décrit avec insistance et beaucoup de détails : elle le battait, le faisait travailler sans relâche, ne lui donnait rien à manger. Or, fait remarquer l'auteur, autant ce thème est banal dans la littérature européenne, autant il est rare dans les namthar (rnam thar, biographie de saint). Elle en conclut qu'il s'agit de faits réels, donc d'une autobiographie et non pas d'une hagiographie plus ou moins idéalisée.

En 1679, le gouvernement central de Lhasa lança une guerre contre le Ladakh. La thèse tibétaine, en grande partie reprise par les historiens, est que cette guerre avait pour but de mettre fin aux persécutions que subissaient les gelukpa de la part d'un pouvoir royal soutenant les drukpa. Dans son article Nawang Jinpa s'inscrit en faux vis-à-vis de cette théorie, faisant remarquer que les rois du Ladakh avaient une politique équilibrée envers les différentes écoles bouddhiques. En cela elle rejoint Rob Linrothe et son analyse du chorten de la reine. Pour elle, il s'agissait tout simplement d'une guerre de conquête, une guerre qui permit au gouvernement de Lhasa d'arracher le Tibet de l'Ouest (Ngaris Skorsum ; mnga' ris skor gsum) au Ladakh. Les Tibétains auraient même conquis le Ladakh si les Moghols n'avaient envoyé des troupes à la rescousse de ce dernier.

Nous parvenons maintenant au xix siècle. John Bray décrit les relations entre le missionnaire morave Heinrich August Jäschke (1817-1883), l'auteur du célèbre TibetanEnglish Dictionnary (Jäschke 1881), et l'un de ses informateurs, Lobsang Chospel (blo bzang chos phel), un moine de Tashi Lhunpo. Sur le plan intellectuel la collaboration fut très fructueuse et Lobsang Chospel s'intégra bien à la mission morave pendant son séjour. Toutefois, sur le plan religieux, chacun des deux hommes resta sur ses positions, l'un attaché à sa foi chrétienne et l'autre à ses croyances bouddhiques.

7 Janet Rizvi fait le portrait de cinq hommes qu'elle a rencontrés dans les années 1980, mais qui étaient actifs dans la première moitié $d u x^{e}$ siècle. Ils pratiquaient alors des professions différentes: un joueur de hautbois (surna), deux forgerons, un médecin traditionnel (amchi), et un "coureur de poste" (mail runner). Les trois premières professions sont connues: elles se pratiquent toujours. La quatrième est plus intéressante car elle a disparu. Sur la route Srinagar-Leh, tous les 4,5 miles (environ $7 \mathrm{~km}$ ), il y avait un relais. Le facteur-coureur y parvenait avec le courrier et immédiatement, quel que soit l'heure, un autre partait jusqu'au relais suivant et ainsi de suite.

Nous voilà parvenus à l'époque actuelle. L'article de Sophie Day, à travers la description de trois membres d'une famille, cherche à cerner l'évolution récente en matière de parenté, d'héritage et de propriété : fin du droit de primogéniture, partage des terres, accès aux emplois salariés, etc., et les difficultés que cela implique : le partage de la 
terre conduit au morcellement des propriétés agricoles qui sont de moins en moins rentables. Cela oblige les membres de la famille à trouver des emplois salariés.

Depuis 1947 le Ladakh est intégré dans l'Union indienne, un pays qualifié par sa constitution de «République souveraine, socialiste, laïque, démocratique (sovereign socialist secular democratic republic)». Néanmoins tout ce qui relève de croyances religieuses, astrologiques, en tout cas peu laïques, résiste toujours. Fonctionnaires comme ONG sont obligés de s'y adapter. Telle est la conclusion de l'article d'Andrea Butcher. Par exemple lorsque les membres d'une ONG doivent participer à une cérémonie religieuse qui doit permettre d'assurer le succès de leur entreprise, laquelle n'a rien de religieux; ou lorsque le gouvernement autonome local (le Hill Council) doit financer en partie la construction d'une grande statue de Maitreya-Chamba (byams pa). La population du gros village de Padum, aujourd'hui chef-lieu du sous-district (tehsil) du Zanskar, est divisée entre bouddhistes et sunnites, à peu près à part égale. En prenant pour fil directeur l'attitude des musulmans lors des trois visites du dalai lama au Zanskar en 1980, 1988 et 2009, Salomé Deboos constate une radicalisation des musulmans qui participèrent avec les bouddhistes, en 1980 et 1988, aux préparatifs de la venue et à l'accueil du dalai lama et ne le firent plus en 2009.

11 Maintenant nous passons au Kinnaur, une vallée au sud du Ladakh, dans l'État de l'Himachal Pradesh. Elle conduit au Shipki la $(5669 \mathrm{~m})$, poste frontière avec la Chine. Rafal Beszterda raconte la fortune des caravanes sur cette route jusqu'au milieu du $\mathrm{xx}^{\mathrm{e}}$ siècle. Après l'attaque de l'Inde par la Chine en 1962, toutes les frontières entre les deux pays furent fermées, puis il fut décidé de rouvrir quelques postes, dont le Shipki la le 16 juillet 1994. Cette route n'a pas retrouvé pour autant sa splendeur d'antan et sa réouverture est plutôt de l'ordre du symbolique. Depuis 1994 quelques Indiens ont franchi le col, mais aucun Tibétain ni Chinois. La Chine est plus intéressée à intégrer le Tibet dans le reste du pays, en particulier par le biais de construction de routes, et l'Inde ne semble pas vouloir faire les efforts nécessaires en matière d'infrastructures.

Enfin Pascale Dollfus analyse la conception que les Ladakhi ont de la couleur. Quelles sont les couleurs de base et les couleurs considérées comme dérivées? Elle étend ensuite l'enquête aux peintures religieuses et finalement conclut que la couleur est rarement un critère mis en avant. Pour donner un exemple, à l'automne on dit que les feuilles du peuplier sont prêtes à tomber plutôt que de dire qu'elles sont jaunes.

\section{BIBLIOGRAPHIE}

Faggionato, C. 2013 A Religious History of the Nubra Valley. Thèse de doctorat (Turin, Université de Turin).

Jäschke, H. A. [1881] 1968 A Tibetan-English Dictionnary (Londres, Routledge/Kegan Paul).

Nawang Tsering 1979 Buddhism in Ladakh. A Study of the Life and Works of the Eighteenth Century Ladakhi Saint Scholar (Londres, Sterling). 
NOTES

1. Pour en savoir plus sur ce célèbre yog, voir Nawang Tsering 1979. 